\title{
Is optic never fibre mis-routing a feature of congenital stationary night blindness?
}

\author{
T. Ung · L. E. Allen · A. T. Moore $\cdot$ D. Trump • \\ I. Zito $\cdot$ A. J. Hardcastle $\cdot$ J. Yates $\cdot$ \\ K. Bradshaw
}

Published online: 13 July 2006

(C) Springer Science+Business Media B.V. 2006

In Documenta Ophthalmologica (2005) 111: 169-178 the article 'Is optic never fiber mis-routing a feature of congenital stationary night blindness?' has been published. Unfortunately, the list of authors was incomplete. Please find the complete list including affiliations here:

T. Ung ${ }^{1}$, L.E. Allen ${ }^{1}$, A.T. Moore ${ }^{1,2}$, D. Trump ${ }^{3,4}$, I. Zito ${ }^{5}$, A.J. Hardcastle ${ }^{5}$, J. Yates ${ }^{3}, \&$ K. Bradshaw ${ }^{1}$ ${ }^{1}$ Ophthalmology Department, Addenbrooke's Hospital, Cambridge, CB2 2QQ, UK;

${ }^{2}$ Moorfields Eye Hospital, London, UK;

${ }^{3}$ Genetics Department, Addenbrooke's Hospital, Cambridge, UK;

${ }^{4}$ Clinical Genetics, University of Manchester, Manchester, UK;

${ }^{5}$ Division of Molecular and Cellular Neuroscience, Institute of Ophthalmology, University College, London, UK

The online version of the original article can be found at http://dx.doi.org/10.1007/s10633-005-5503-9

T. Ung · L. E. Allen · A. T. Moore · K. Bradshaw ( $\square)$ Ophthalmology Department, Addenbrooke's Hospital, Cambridge CB2 2QQ, UK

e-mail: keith.bradshaw@addenbrookes.nhs.uk

\section{A. T. Moore}

Moorfields Eye Hospital, London, UK

D. Trump $\cdot$ J. Yates

Genetics Department, Addenbrook's Hospital,

Cambridge, UK

D. Trump

Clinical Genetics, University of Manchester,

Manchester, UK

I. Zito · A. J. Hardcastle

Division of Molecular and Cellular Neuroscience, Institute of Ophthalmology, University College,

London, UK 